\title{
Sistemas de Predicción por Conjuntos en el mundo, proyectos TIGGE y TIGGE-LAM
}

\author{
JUANMA SANCHO ÁVILA \\ Centro Nacional de Predicción (CNP), Agencia Estatal de Meteorología (AEMET)
}

No vayas a creer lo que te cuentan del mundo (ni siquiera esto que te estoy contando), ya te dije que el mundo es incontable.

MARIO BENEDETTI

El experimento de investigación de sistemas de observación y predecibilidad, en inglés The Observing System Research and Predictability Experiment (THORPEX), es un proyecto de la Organización Meteorológica Mundial, World Meteorological Organization (WMO), cuyo objetivo es mejorar las predicciones de fenómenos adversos de 1 día a 2 semanas. Dentro de THORPEX existe un proyecto interactivo de sistema(s) de predicción por conjuntos (SPC) globales, el proyecto THORPEX Interactive Grand Global Ensemble (TIGGE), que nació en 2005 con el fin de crear un archivo de acceso a la comunidad científica en el que estuvieran disponibles los datos de los modelos atmosféricos globales producidos por los nueve centros principales del mundo. El objetivo fundamental es que los investigadores puedan disponer de los datos almacenados para comparar los diferentes SPC con el fin de entender más profundamente la influencia de las diferentes fuentes de error en la contribución final a la calidad de las predicciones según los diferentes diseños de los SPC. Otro objetivo importante es estudiar e investigar las posibilidades de mejora en el desarrollo de SPC combinando diferentes ensembles en los llamados enfoques multimodelo. En este técnico capítulo, por un lado, se describen en detalle los proyectos TIGGE y TIGGE-LAM. Por otro lado, se revisan los principales SPC del mundo, participen en TIGGE o no, lo que permite esbozar una silueta del estado del arte a nivel mundial en la actualidad.

Palabras clave: sistemas de predicción por conjuntos en el mundo, proyecto TIGGE, proyecto TIGGE-LAM. 


\subsection{SPC globales en el mundo: EI proyecto TIGGE}

Fueron EPSTEIN [7] y LeITH [18] los pioneros en proponer un enfoque basado en predicciones probabilistas en lugar del uso de modelos deterministas a la vista de la rápida degradación de la calidad de los pronósticos con el alcance de la predicción.

Pero no fue hasta diciembre de 1992, después de una investigación exhaustiva acerca de cómo perturbar de forma efectiva las condiciones iniciales y la física de los modelos de predicción, cuando el European Centre for Medium-range Weather Forecasts -Centro Europeo de Predicción a Plazo Medio- (ECMWF) (cap. 19 en la página 289) y el National Centers for Environmental Prediction, EE. UU. (NCEP) iniciaron la producción de predicciones probabilistas de forma operativa. A estos siguieron poco después el canadiense MSC (Meteorological Service of Canada) en 1995, y más tarde otros seis centros nacionales comenzaron a implementar sistema(s) de predicción por conjuntos (SPC): el australiano BMRC (Australian Bureau of Meteorology), el chino CMA (China Meteorological Administration), el brasileño CPTEC (Brazilian Center for Weather Prediction and Climate Studies), el japonés JMA (Japanese Meteorological Administration), el coreano KMA (Korean Meteorological Administration) y el británico UKMO (United Kingdom Meteorological Office).

A estos nueve SPC, que contribuyen al proyecto TIGGE y que actualmente proporcionan de forma operativa productos basados en predicciones probabilistas a escala global, habría que añadir también el SPC global de Météo-France, denominado PEARP (Prévision d'Ensemble ARPEGE). Por otro lado el DWD alemán está actualmente desarrollando un sistema de asimilación para su modelo a escala global ICON (Icosahedral Nonhydrostatic Model), basado en asimilación de datos por conjuntos (Ensemble of Data Asimilation (EDA), sec. 16.2.5 en la página 248 [15, 16]) y un SPC a escala global (ICON-EPS).

Anteriormente, la información producida diariamente por los SPC de los diferentes Centros Nacionales de Predicción podía ser accedida únicamente desde cada centro. Además, el formato de los datos producidos así como los campos, variables e incluso unidades eran distintos para cada sistema.
El experimento de investigación de sistemas de observación y predecibilidad, en inglés The Observing System Research and Predictability Experiment (THORPEX), es un proyecto de la Organización Meteorológica Mundial, World Meteorological Organization (WMO), cuyo objetivo es mejorar las predicciones de fenómenos adversos de 1 día a 2 semanas.

Dentro de THORPEX existe un proyecto interactivo de SPC globales, el proyecto THORPEX Interactive Grand Global Ensemble (TIGGE), que nació en 2005 con el fin de crear un archivo de acceso para la comunidad científica en el que estuvieran disponibles los datos producidos por los 9 centros en un formato homogéneo, estándar y con las mismas unidades. El objetivo fundamental era que los investigadores pudieran utilizar los datos almacenados para comparar los diferentes SPC con el fin de entender más profundamente la influencia de las diferentes fuentes de error en la contribución final de la calidad de las predicciones según los diferentes diseños de los SPC. Otro objetivo importante era estudiar e investigar las posibilidades de mejora en el desarrollo de SPC combinando diferentes ensembles en los llamados enfoques multimodelo.

En TIGGE se almacenan diariamente, cada 6 horas, a nivel global y para todo el alcance de la predicción, campos superficiales de 30 variables así como campos en altura a varios niveles de la atmósfera para distintas variables. La disponibilidad de los datos en TIGGE se remonta a octubre de 2006 (dependiendo el inicio del archivo de los datos de cada centro nacional de predicción). El acceso a los datos almacenados en TIGGE se puede hacer a través de tres centros mundiales: el ECMWF europeo, el CMA chino y el National Center for Atmospheric Research (NCAR) en Estados Unidos.

Una de las conclusiones de la implementación del archivo TIGGE es que no existe una receta única para el tratamiento de las fuentes de error en el diseño de SPC. Es posible utilizar distintos enfoques y tratamientos aunque, como se verá posteriormente, hay sistemas que muestran claramente un mejor comportamiento que otros, como es el caso del sistema del ECMWF, que hoy por hoy se comporta con claridad mejor que el resto, sobre todo para variables en escala sinóptica y a medio plazo. 


\begin{tabular}{ccccccc}
\hline Centro & \multirow{2}{*}{ \#M } & Pert. CCII & Pert. Mod & Alcance & $\begin{array}{c}\text { Resolución } \\
\text { horiz. }(\mathrm{km}) / \text { vert. (\#niveles) }\end{array}$ & \#Ejecuciones \\
\hline ECMWF (EU) & $1+50$ & SV +EDA & SPPT+ SKEB & $0-15 \mathrm{~d}$ & TCO639 $(18 \mathrm{~km}) / 91$ & $00 / 12$ \\
& & & & $\begin{array}{c}16-46 \mathrm{~d} \\
\text { TCO319 }(36 \mathrm{~km}) / 91 \text { niveles }\end{array}$ & \\
NCEP (EEUU) & $1+20$ & ETR & SPPT & $0-7 \mathrm{~d}$ & T254 $(90 \mathrm{~km}) / 28$ niveles & $00 / 06 / 12 / 18$ \\
& & & & $8-16 \mathrm{~d}$ & T190 $(120 \mathrm{~km}) / 28$ niveles & \\
CMA (China) & $1+14$ & BV & NO & $0-10 \mathrm{~d}$ & T213(70 km)/31 niveles & $00 / 12$ \\
CMC (Canada) & $1+20$ & EnKF & SPPT+ SKEB & $0-16 \mathrm{~d}$ & $600 \times 300(75 \mathrm{~km}) / 40$ niveles & $00 / 12$ \\
UKMO (UK) & $1+17$ & ETKF & RP2+ SKEB & $0-7 \mathrm{~d}$ & N640 $(20 \mathrm{~km}) / 70$ niveles & $00 / 06 / 12 / 18$ \\
BMRC (Au) & $1+32$ & SV & NO & $0-10 \mathrm{~d}$ & TL119(210 km) $/ 19$ niveles & $00 / 12$ \\
CPTEC (Br) & $1+14$ & EOF & NO & $0-15 \mathrm{~d}$ & T126(120 km)/28 niveles & $00 / 12$ \\
JMA (Jap) & $1+25$ & SV+ETKF & SPPT & $0-11 \mathrm{~d}$ & T479 $(50 \mathrm{~km}) / 100$ niveles & $00 / 12$ \\
KMA (Kor) & $1+23$ & ETKF & RP2+ SKEB & $0-12 \mathrm{~d}$ & N320 $(40 \mathrm{~km}) / 70$ niveles & $00 / 06 / 12 / 18$
\end{tabular}

Tabla 26.1: Principales Características de los 9 modelos en TIGGE en agosto 2017. \#M: Número de miembros perturbados $(1+$ indica un miembro adicional de control, no perturbado). Perturbaciones condiciones iniciales (CCII): Método de perturbación: Bred Vectors (BV [25], Ensemble Kalman filter (EnKF [14]), Ensemble Transform Kalman Filter (ETKF) [2, 26], Ensemble Transform with Rescaling (ETR [27]); EDA [3, 4, 16]; Singular Vectors (SV [22]); Empirical Orthogonal Functions (EOF [21]). Perturbaciones en el modelo (Mod): Stochastic Perturbed Parameterization Tendencies (SPPT) [5]; Stochastic Kinetic Energy Backscatter (SKEB) [1]; Random Parameters $(R P 2,[23])$

\subsubsection{Características de los Sistemas de Predicción por Conjuntos (SPC)}

En la actualidad se siguen archivando los datos de 7 sistemas de predicción global en la base de datos de TIGGE, ya que los procedentes del modelo Australiano (BMRC) dejaron de archivarse en julio de 2010 y los del modelo británico (UKMO) en julio de 2014. Los diferentes SPC están desarrollados con el fin de simular el efecto de las principales fuentes de error en las predicciones numéricas. Por un lado, las incertidumbres en las condiciones iniciales propias, debidas tanto a los errores observacionales como a las aproximaciones realizadas en el proceso de asimilación de datos para la estimación estado inicial, también llamado análisis de la atmósfera. Por otro lado, las incertidumbres del modelo debidas tanto a las aproximaciones de las ecuaciones de la física del mismo como a las simplificaciones asociadas a las parametrizaciones de los procesos físicos en resoluciones mayores que la malla del modelo. Los enfoques para el tratamiento de estas fuentes de error son diferentes aunque comunes en algunos casos (ver Tabla 26.1). Una ejecución del modelo o miembro de control, se alimenta inicialmente por un análisis no perturbado de la atmósfera durante el proceso de asimilación de datos, de manera que los datos observacionales son introducidos en el modelo sin ser añadidas perturbaciones. El resto de integraciones o predicciones, que constituyen el conjunto de los llamados miembros perturbados, simulan los efectos de las incertidumbres en la medida y asimilación de las observaciones. La mayor parte de ellos, además, tiene también en cuenta las incertidumbres o errores propios de la física del modelo a través de la aplicación de perturbaciones estocásticas a las tendencias del mismo. También hay diferencias sustanciales en el número de miembros perturbados, la resolución tanto horizontal como en número de niveles verticales, el alcance máximo de predicción y el número de ejecuciones diarias de los sistemas, que por lo general suelen ser dos $(00 \mathrm{~h} \mathrm{y}$ $12 \mathrm{~h})$.

Con el fin de estimar el coste computacional en la producción de los ensembles por parte de cada uno de los centros, es útil definir los siguientes cuatro índices:

- La resolución espacial, $H R^{2}$ (que tiene en cuenta que si la resolución se duplica, el número de puntos de grid se multiplica por 4).

- El número de niveles verticales $L E V$

- El número de miembros por día \#M

- El alcance de la predicción en días $F C D$. 


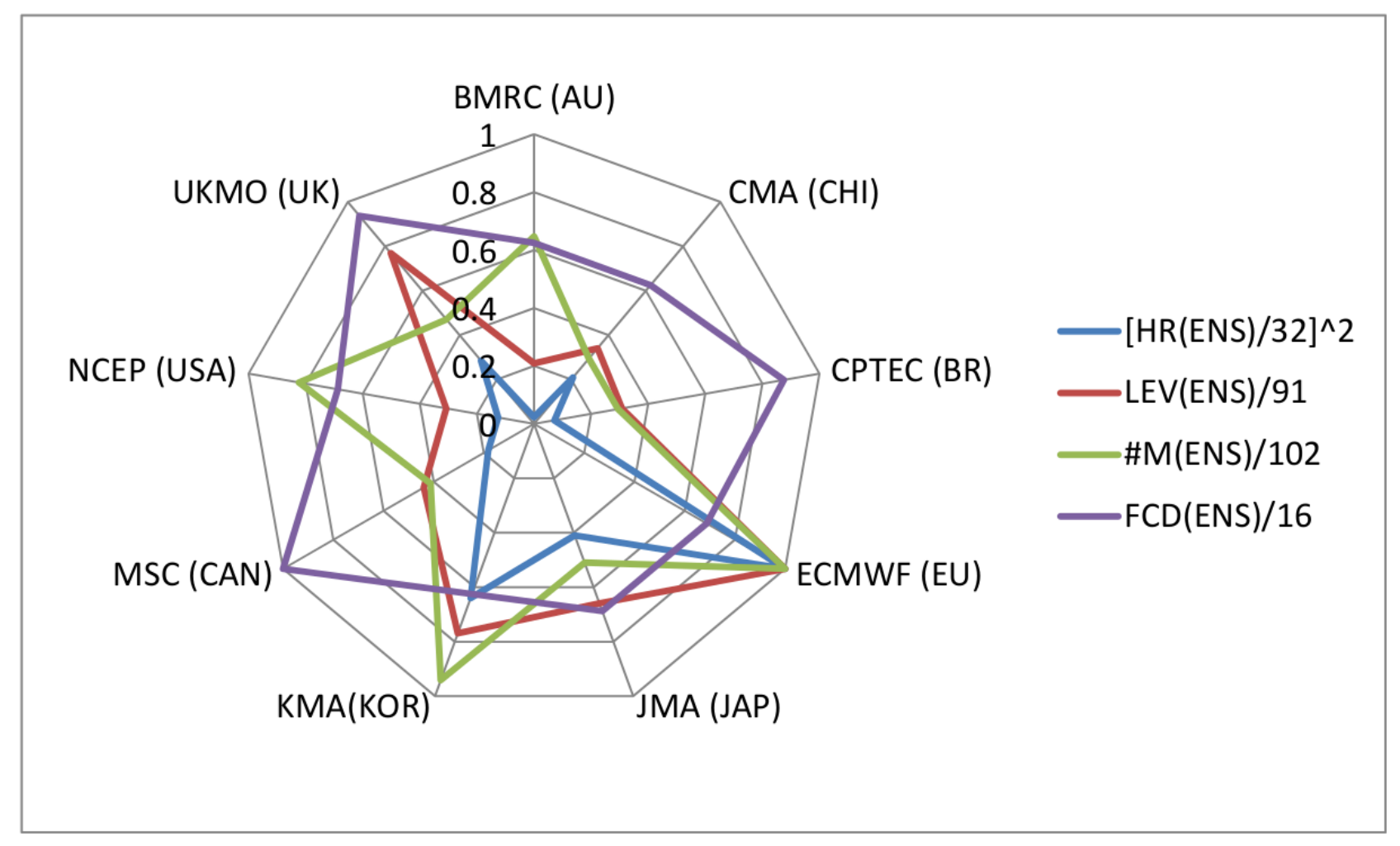

Figura 26.1: Comparación de 4 índices normalizados de coste computacional: el cuadrado de la resolución horizontal (HR), el número de niveles verticales (LEV), el número de miembros perturbados por día (\#M) y el alcance de la predicción en días (FCD). Cada índice esta normalizado con respecto al máximo valor de entre todos los SPC (ECMWF, 2017).

Estos cuatro índices están normalizados dividiendo los valores por el correspondiente al SPC que proporciona el valor mas costoso computacionalmente (Ver Figura 26.1). El esfuerzo o coste computacional relativo $r_{n}$ en la producción de cada predicción probabilista diaria en cada SPC se puede estimar con el producto de estos cuatro índices:

$r_{n}=\left(H R_{n} / 32\right)^{2} \cdot\left(L E V_{n} / 91\right) \cdot\left(\# M_{n} / 102\right) \cdot\left(F C D_{n} / 16\right)$

Teniendo en cuenta este índice, es el SPC del ECMWF el que requiere más recursos computacionales debido fundamentalmente a su mayor número de miembros y resolución tanto horizontal como en número de niveles verticales, seguido por el SPC coreano del KMA, el japonés del JMA, el británico del UKMO, el canadiense del MSC y el resto de sistemas. Existe una relación directa entre los recursos computacionales necesarios para la producción de predicciones probabilistas y la calidad de dichas predicciones.

A continuación se describirán brevemente las carac- terísticas principales de los distintos SPC a escala global.

\subsubsection{Europa, ECMWF (European Centre for Medium-Range Weather Fore- casts), ECENS}

El SPC del ECMWF, llamado ECENS (ECmwf Ensemble Prediction System), fue uno de los dos sistemas pioneros junto con el GEFS (Global Ensemble Forecast System) del NCEP norteamericano, en la producción de predicciones probabilistas a escala global a partir de noviembre de 1992. Se comenzó a archivar información del ECENS en TIGGE en octubre de 2006, continuando en la actualidad. Sus características actuales (2018) son las siguientes:

- Está formado por 51 miembros (1 de control y 50 perturbados) 
- Se ejecuta 2 veces al día (00 h/12 h) con un alcance de 46 días y una resolución variable según el alcance de la predicción

- La resolución horizontal es de $18 \mathrm{~km}$ hasta los 15 días y de $36 \mathrm{~km}$ desde los 16 a los 46 días de alcance. El número de niveles en la vertical es de 91 en ambos casos. Anteriormente la resolución horizontal disminuía a partir del día 10 y es a partir de la introducción del ciclo 41r2 en marzo de 2016 cuando se introducen estas importantes mejoras en la resolución horizontal.

- El modelo atmosférico esta acoplado con un modelo dinámico oceánico (NEMO) con una resolución horizontal de 1 grado y 42 capas oceánicas y a un modelo de olas (WAM) con $55 \mathrm{~km}$ de resolución. Esta es una mejora destacable con respecto a otros sistemas de predicción, sobre todo para los alcances mayores. El acoplamiento con el modelo oceánico se realiza desde noviembre de 2013 desde el alcance mas corto y no desde el día 10 como anteriormente.

- Las condiciones iniciales son perturbadas utilizando combinaciones lineales de Vectores Singulares (Singular Vectors, SV [22], sec. 16.2.6 en la página 249) y perturbaciones definidas por asimilación de datos por conjuntos (EDA [15, 16], sec. 16.2.5 en la página 248). Los vectores singulares se calculan teniendo en cuenta las direcciones en las que la energía asociada a las perturbaciones crecen más rápidamente en un intervalo de $48 \mathrm{~h}$. Por otra parte, cada uno de los 25 miembros EDA se genera perturbando las observaciones según una gaussiana con una desviación estándar de acuerdo con la muestra de observaciones reales y haciendo evolucionar cada trayectoria en una ventana de asimilación de $12 \mathrm{~h}$ teniendo en cuenta los errores del modelo según un esquema SPPT [5]. Una vez calculados los vectores singulares, estos se combinan con los miembros EDA para obtener 25 miembros perturbados. Los otros 25 miembros que faltan hasta alcanzar los 50 miembros que constituyen el ensemble completo se obtienen cambiando el signo a dichos miembros obteniendo, por tanto, otras 25 perturbaciones simétricas.

- La incertidumbre del modelo atmosférico se tiene en cuenta mediante la introducción de perturbaciones estocásticas de las tendencias SPPT y el método SKEB [1]. El SPPT está diseñado para simular errores aleatorios del modelo debido a los procesos físicos parametrizados y el método SKEB simula la transferencia de energía desde escalas no resueltas por el modelo a escalas que sí lo son, es decir, de fenómenos de resoluciones finas a escalas mayores.

\subsubsection{EEUU, NCEP (National Centers of Environmental Prediction, USA), GEFS}

El sistema estadounidense, denominado GEFS (Global Ensemble Forecast System), comenzó a funcionar operativamente en diciembre de 1992, disponiéndose de datos en TIGGE desde 2007. Las predicciones probabilistas de los sistemas NCEP y del CMC canadiense son intercambiadas en tiempo real generando un sistema de predicción por conjuntos multimodelo para América del Norte en el marco del proyecto NAEFS (North American Ensemble Forecast System), en el que además de Estados Unidos y Canadá, está involucrado el servicio meteorológico mexicano. Las características de este sistema son las siguientes:

- Esta formado por 21 miembros (1 miembro de control y 20 perturbados).

- Se ejecuta 4 veces al día (00/06/12/18) con un alcance de 16 días.

- La resolución horizontal es variable según el alcance de las predicciones: $90 \mathrm{~km}$ hasta 7 días y $120 \mathrm{~km}$ hasta 16 días, siendo 28 los niveles verticales.

- No cuenta con un modelo oceánico acoplado al atmosférico.

- El esquema de perturbaciones de las condiciones iniciales esta basado en un esquema Ensemble Transform with Rescaling (ETR [26]) que es una evolución del método original Bred Vectors (BV $[24,25])$.

- Las incertidumbres del modelo están tratadas según un esquema SPPT.

\subsubsection{China, CMA (China Meteorological Administration)}

El sistema desarrollado por China comenzó en 2001 continuando en la actualidad, archivando datos en TIGGE desde 2007.

Sus características principales son las siguientes:

- Consta de 15 miembros (1 miembro de control y 14 perturbados).

- Se ejecuta diariamente hasta 10 días a las $0 \mathrm{~h}$ y $12 \mathrm{~h}$.

- Su resolución horizontal es de $70 \mathrm{~km}$ con 31 niveles verticales. 
- Solo tiene en cuenta las componentes de tierra y atmosférica, ya que no cuenta con un modelo oceánico acoplado al atmosférico.

- El método de perturbación de las condiciones iniciales se basa en Bred Vectors (BV) basados en la primera versión del ensemble del NCEP (EE. UU.).

- No se simulan incertidumbres de la física del modelo, es decir, se hace la suposición de modelo perfecto.

\subsubsection{Canadá, CMC (Meteorological Cen- tre of Canada), GEPS}

Denominado GEPS (Global Ensemble Prediction System), el sistema canadiense inició la producción de predicciones probabilistas en 1998, estando disponibles los datos en TIGGE desde 2007. Este sistema:

- Consta de 21 miembros (1 miembro de control y 20 perturbados).

- El alcance es de 16 días con 2 ejecuciones diarias (00/12).

- La resolución horizontal es de $75 \mathrm{~km}$ con 40 niveles en la vertical.

- No cuenta con un modelo oceánico acoplado al atmosférico.

- El método de perturbación de las condiciones iniciales se basa en un esquema Ensemble KALMAN Filter (EnKF).

- La incertidumbre del modelo atmosférico se estima mediante la introducción de perturbaciones estocásticas a las tendencias SPPT y el método SKEB.

\subsubsection{Reino Unido, UKMO (United Kingdom Meteorological Office), MOGREPS-G}

Este sistema se describe con mayor detalle en el capítulo 23 en la página 361. No obstante, se describe aquí por completitud en el capítulo. El sistema desarrollado en Met Office, denominado MOGREPS-G (Met Office Global and Regional Ensemble Prediction System Global version, cap. 23 en la página 361), comenzó a producir predicciones en 2005, interrumpiendo su operación en 2014 con el fin de continuar más adelante generando conjuntos de predicciones siguiendo un enfoque basado en Lagged Average Forecasts, LAF $[6,13,20]$. Los datos están disponibles en TIGGE desde 2006.
- Está formado por 18 miembros (1 miembro de control y 17 perturbados).

- El alcance es de 7 días con 4 ejecuciones diarias (00/06/12/18 h).

- La resolución horizontal es de $20 \mathrm{~km}$ con 70 niveles verticales.

- No cuenta con un modelo oceánico acoplado al atmosférico.

- Para la generación de las perturbaciones en las condiciones iniciales utiliza un esquema ETKF.

- La incertidumbre en el modelo esta simulado mediante la introducción de parámetros aleatorios RP2 y el método SKEB.

\subsubsection{Australia, BMRC (Bureau of Me- teorology Research Center), BoM- EPS}

El SPC del BMRC, denominado BoM-EPS (BMRC Medium-Range Ensemble Prediction System), comenzó a producir predicciones por conjuntos en julio de 2001 y ha continuado hasta julio de 2010, momento en que cesó la producción con el fin de comenzar de nuevo después de desarrollar un sistema de asimilación de datos y de predicción similar al sistema del UKMO.

Las características de este sistema no han variado sustancialmente desde su inicio en 2001 hasta 2010 y son las siguientes:

- Está formado por 33 miembros (1 miembro de control y 32 perturbados).

- El alcance de predicción es de 10 días con 2 ejecuciones diarias (00 UTC / 12 UTC).

- La resolución horizontal es de aproximadamente $210 \mathrm{~km}$ con 19 niveles verticales.

- No tiene en cuenta un modelo oceánico acoplado al modelo atmosférico.

- El esquema de perturbación de las condiciones iniciales se basa en la primera versión de Vectores Singulares desarrollado en el ECMWF y son computados solo para el Hemisferio Sur.

- No se simulan incertidumbres de la física del modelo.

\subsubsection{Brasil, CPTEC (Centro de Previ- sión del Tiempo y Estudios Climá- ticos)}

El CPTEC comenzó a producir predicciones probabilistas en 2001 y se archivan en TIGGE desde 2008. 
Su sistema:

- Consta de 15 miembros (1 miembro de control y 14 perturbados).

- Produce predicciones hasta 15 días con 2 ejecuciones diarias (00 UTC / 12 UTC).

- La resolución horizontal es de $120 \mathrm{~km}$ con 28 niveles en la vertical.

- No considera un modelo oceánico acoplado al modelo atmosférico.

- Las incertidumbres en las condiciones iniciales se obtienen a partir de un sistema basado en funciones empíricas ortogonales (Empirical Orthogonal Functions; EOF [19, 21]). El miembro de control se obtiene a partir del análisis del NCEP.

- No utiliza un esquema de errores del modelo.

\subsubsection{Japón, JMA (Japan Meteorological Agency)}

El SPC de Japón comenzó a funcionar de manera operativa desde 2001 y archiva datos en TIGGE desde 2011. Este sistema:

- Consta de 26 miembros (1 miembro de control y 25 perturbados).

- Produce predicciones hasta 11 días con 2 ejecuciones diarias (00 UTC / 12 UTC).

- La resolución horizontal es de 50 km con 100 niveles en la vertical.

- No considera un modelo oceánico acoplado al modelo atmosférico.

- Las incertidumbres en las condiciones iniciales se obtienen a partir del un sistema basado en funciones empíricas ortogonales EOF. El miembro de control se obtiene a partir del análisis del NCEP.

- Las condiciones iniciales son perturbadas utilizando combinaciones lineales de Vectores Singulares (SV) y recientemente (2017) también mediante el método basado en ETKF.

- La incertidumbre del modelo atmosférico se tiene en cuenta mediante la introducción de perturbaciones estocásticas a las tendencias SPPT. Sistema similar al originalmente desarrollado en el ECMWF.

\subsubsection{Corea, KMA (Korea Meteorological Agency)}

El sistema coreano comenzó a funcionar de manera operativa en marzo de 2000, archivando datos en TIG-
GE desde 2007. Desde el año 2011 utiliza un sistema muy similar al SPC del UKMO. Sus características son:

- Está formado por 24 miembros (1 miembro de control y 23 perturbados)

- Se realizan predicciones con un alcance de hasta 12 días con 4 pasadas diarias $(00 / 06 / 12 / 18 \mathrm{~h})$

- La resolución horizontal es de $40 \mathrm{~km}$ y 70 niveles verticales

- No tiene en cuenta un modelo oceánico ni de olas acoplado al modelo atmosférico

- Las condiciones iniciales se perturban utilizando el esquema ETKF.

- La incertidumbre del modelo se tiene en cuenta mediante la introducción de parámetros aleatorios RP2 y el método SKEB.

\subsubsection{Francia, Météo-France, PEARP}

Météo-France comenzó a ejecutar en 2004 su sistema de predicción por conjuntos denominado PEARP (Prévision d'Ensemble ARPEGE), basado en su modelo determinista a escala global ARPEGE (Action de Recherche Petite Echelle Grande Echelle). A diferencia del resto de SPC globales, se caracteriza por utilizar una resolución espacial variable, de forma que sobre el dominio de Francia la resolución es mayor que en el resto del globo.

- Consta de 35 miembros (1 miembro de control y 34 perturbados).

- El alcance de las predicciones es de 4.5 días con una única pasada a las $18 \mathrm{~h}$.

- La resolución espacial es variable: $15.5 \mathrm{~km}$ sobre Francia y $89 \mathrm{~km}$ en el resto del globo, contando con 65 niveles en la vertical.

- Las condiciones iniciales son perturbadas utilizando Vectores Singulares (SV) y perturbaciones definidas por un conjunto de asimilación de datos, EDA.

- Para tener en cuenta el error del modelo se realiza un enfoque multifísica basado en 10 configuraciones distintas de parametrizaciones físicas. 


\begin{tabular}{ccccc}
\hline Centro & \#Miembros & Miembro de control & Alcance $(\mathrm{h})$ & Ejecuciones diarias \\
\hline ALADIN-LAEF & 16 & YES & 72 & $00 / 12$ \\
COSMO-DE-EPS & 20 & NO & 27 & $00 / 03 / 06 / 09 / 12 / 15 / 18 / 21$ \\
COSMO-LEPS & 16 & NO & 132 & $00 / 12$ \\
DMI-HIRLAM & 25 & YES & 64 & $00 / 06 / 12 / 18$ \\
GLAMEPS & 54 & NO & 60 & $06 / 18$ \\
HUNEPS & 11 & YES & 60 & 18 \\
MOGREPS & 12 & NO & 54 & $03 / 09 / 15 / 21$ \\
PEARP & 35 & YES & 54 & $06 / 18$ \\
\hline
\end{tabular}

Tabla 26.2: Principales Características de los ocho modelos presentes en TIGGE-LAM en agosto de 2017.

\subsection{SPC regionales: Proyecto TIGGE-LAM}

El proyecto TIGGE-LAM es una extensión del archivo TIGGE para incluir información procedente de SPC regionales desarrollados utilizando modelos de área limitada (Limited Area Models; LAM). Estas predicciones probabilistas se producen para resoluciones de 2 a $12 \mathrm{~km}$ y para alcances de unos pocos días.

El proyecto TIGGE-LAM permite mejorar las metodologías para la aplicación y generación de SPC regionales y comparar los diferentes sistemas para el corto o muy corto plazo. Inicialmente los métodos de predicción probabilistas estaban enfocados al medio plazo y en los últimos años se está avanzando en el desarrollo y uso de estos métodos en el corto y muy corto plazo.

Por otra parte, el archivo TIGGE-LAM proporciona una información muy útil para el desarrollo de los sistemas globales de predicción por conjuntos descritos anteriormente, ya que estos están evolucionando rápidamente hacia resoluciones mayores y es previsible que lo sigan haciendo en los próximos años.

Los ensembles basados en modelos de área limitada se desarrollan para resoluciones mayores (actualmente de $2 \mathrm{~km}$ a $25 \mathrm{~km}$ ), y alcances menores (de 18 a $72 \mathrm{~h}$ ) que en el caso de los SPC globales descritos anteriormente. Cuando un modelo puede resolver explícitamente la convección (debido a sus características y su configuración en alta resolución), es capaz de representar de forma más realista los típicos patrones de los campos de precipitación, por ejemplo. Sin embargo, las predicciones de la convección (así como de otros procesos que tienen lugar a pequeña escala) están muy limitados por su predecibilidad determinista, que es inevitablemente baja debido a su comportamiento caótico. Por tanto, incluso para un plazo corto de solo 24 horas, la predicción de detalles de la convección como la localización o el momento en que se puede producir una tormenta es normalmente incierta.

Los SPC basados en modelos de área limitada y alta resolución pueden añadir información a los pronósticos procedentes de modelos de alta resolución deterministas. Por esta razón, muchos servicios meteorológicos nacionales están desarrollando sistemas regionales de predicción por conjuntos.

\subsubsection{Características específicas}

Los sistemas regionales de predicción por conjuntos están basados en modelos de área local no hidrostáticos y de alta resolución. Son desarrollados dentro de diferentes proyectos, como es el caso de ALADIN (desarrollado y mantenido por un consorcio de 16 Servicios Meteorológicos Nacionales liderados por Météo-France), COSMO (desarrollado por un consorcio de 7 Servicios Meteorológicos Nacionales y liderado por el servicio alemán, Deutscher Wetterdienst), Weather Research and Forecasting (WRF) (desarrollado en Estados Unidos por el NCAR, la National Oceanic and Atmospheric Administration (NOAA) y otros), HARMONIE (un modelo que comparte código con ALADIN, y desarrollado por un consorcio de 10 SMN), y el Unified Model (MetOffice).

Estos sistemas pueden tener un ciclo de asimilación de datos que utiliza una extensa variedad de observa- 
ciones meteorológicas, incluyendo en algunos casos datos procedentes de radares meteorológicos, tratando de representar con la máxima fiabilidad posible los patrones de precipitación en las observaciones que alimentan el sistema. Necesitan, así mismo para su ejecución, condiciones de contorno laterales que proceden normalmente de SPC globales con menor resolución.

En la Tabla 26.2 en la página anterior se muestran algunas de las principales características de los SPC regionales que se archivan en TIGGE-LAM.

A modo de ejemplo, en las subsecciones que siguen se resumen las características de los SPC regionales como COSMO-DE-EPS [10] del DWD, AromeFrance-EPS de Météo-France, SREF [25] de NCEP y el AEMET-SREPS [9]. Otros sistemas son el noruego EPS LAMEPS [8], el hungaro LAMEPS basado en ALADIN [11], el sistema multimodelo GLAMEPS [17] (de los consorcios ALADIN y HIRLAM) y la versión en área limitada de MOGREPS [12] (MetOffice).

\subsubsection{COSMO-DE-EPS de COSMO}

El sistema de predicción por conjuntos COSMO-DEEPS entró en operación en 2012 y participa actualmente en el proyecto TIGGE-LAM. Está basado en el modelo no hidrostático (y que por lo tanto puede resolver la convección) COSMO-DE y produce predicciones probabilistas en un grid de $2.8 \mathrm{~km}$ y para 50 niveles verticales en un dominio centrado en Alemania abarcando también algunos países colindantes. Está formado por 20 miembros con un alcance de 27 horas ( 45 horas en el caso de la pasada de las 03 UTC), ejecutándose cada 3 horas. Utiliza un sistema de perturbación de las condiciones iniciales basado en un esquema Ensemble Kalman Filter y condiciones de contorno procedentes de 4 modelos globales (ECMWF, ICON del DWD, GFS del NCEP y GSM de Japón). Sin embargo, está prevista una próxima sustitución de este sistema de perturbación de las condiciones de contorno por un sistema basado en el uso de distintos miembros de un único modelo (ICON), es decir, abandonar el enfoque multimodelo en favor de un sistema monomodelo. También tiene en cuenta el error del modelo mediante un sistema de perturbaciones de un número fijo de parámetros que se distribuyen de manera aleatoria entre los 20 miembros del sistema, manteniéndose posteriormente fijos durante cada ejecución. También es destacable que, durante el proceso de asimilación, se alimenta de estimaciones de datos de precipitación procedentes de radares meteorológicos.

\subsubsection{Arome-France-EPS de Météo- France}

El sistema regional de mesoescala Arome-FranceEPS, desarrollado por Météo-France, entró en funcionamiento en modo de desarrollo en septiembre de 2015 y de forma operativa en octubre de 2016. Está basado en el modelo no hidrostático HARMONIEAROME y consta de 12 miembros con una resolución horizontal de $2.5 \mathrm{~km}$ y 60 niveles en la vertical. Se ejecuta dos veces al día (09/21 UTC) para un alcance de 45 horas. Utiliza un sistema de perturbación de las condiciones iniciales y de contorno basado en un algoritmo de agrupamiento (clustering) de perturbaciones de PEARP (Prévision d'Ensemble ARPEGE), el sistema de predicción por conjuntos a escala global de Météo-France, centrando dichas perturbaciones en torno al análisis determinista del modelo de mesoescala HARMONIE-AROME. Los errores en la física del modelo se tienen en cuenta mediante un esquema de perturbaciones estocásticas de las tendencias, SPPT (sec. 17.4.4 en la página 269).

\subsubsection{Short Range Ensemble Forecast (SREF) del NCEP}

En cuanto al sistema de predicción por conjuntos regional desarrollado por NCEP empezó a funcionar de forma operativa en 2001 y se denomina SREF (Short Range Ensemble Forecast). Este sistema está basado en el modelo no hidrostático de mesoescala WRF y sus diferentes configuraciones, teniendo en cuenta los diferentes tratamientos de las parametrizaciones físicas y el uso de los dos núcleos dinámicos desarrollados para este modelo: el NMN (Non-hydrostatic Mesoscale Model), desarrollado por el NCEP y el ARW (Advanced Research WRF) del NCAR. Está formado por 26 miembros que se ejecutan 4 veces al día $(03 / 09 / 15 / 21$ h) con un alcance de hasta 87 horas para el dominio de Norteamérica. La resolución horizontal es de $16 \mathrm{~km}$ con 40 niveles verticales. Las condiciones de contorno son proporcionadas por el modelo global GFS (Global Forecast System) actualizadas cada 3 horas. Es un sistema multimodelo que trata la incertidumbre en la modelización de la física 
teniendo en cuenta varios modelos (o configuraciones del mismo modelo) diferentes. De forma análoga, las condiciones iniciales se tratan en un enfoque multianálisis y utilizando técnicas LAF (Lagged Average Forecast).

\subsubsection{AEMET-SREPS de AEMET}

El sistema AEMET-SREPS (este sistema se describe con pleno detalle en el cap. 21 en la página 313) funcionó diariamente desde 2007 hasta 2014. Combinaba técnicas multimodelo para los errores en la formulación del modelo, multianálisis para las incertidumbres en las condiciones iniciales y multicondiciones de contorno para el tratamiento de los errores debidos a las condiciones de contorno. Utilizaba 5 modelos globales (DWD-GME, ECMWF-IFS, MSC-CMC,
NCEP-GFS y UKMO) para las condiciones iniciales y de contorno y 4-5 modelos de área limitada (de entre los cinco siguientes: COSMO-LM, DWD-HRM, HIRLAM, MM5 y UKMO-UM) que se ejecutaban para cada una de las condiciones de contorno e iniciales, proporcionando finalmente un total de 20-25 miembros. La resolución horizontal era de $25 \mathrm{~km}$ con alcances de hasta 72 horas ejecutándose 2 veces al día, cubriendo una región amplia que incluía el Atlántico Norte, Europa y el norte de África.

\subsection{Personalidades}

En la Figura 26.2 en la página siguiente se da un pequeño detalle de algunas personalidades responsables del surgimiento y desarrollo de los SPC pioneros en el mundo. 

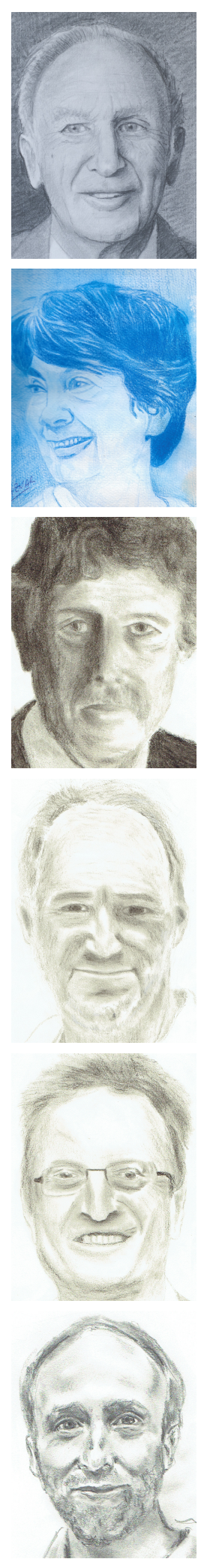

EDWARD NORTON LORENZ (1917-2008), matemático y meteorólogo estadounidense, formuló el concepto de atractor extraño e introdujo el caos en meteorología, acuñando o recuperando (sec. 5.5 en la página 64) la idea de efecto mariposa.

EUGENIA KALNAY, meteoróloga argentina, experta en dinámica y predecibilidad, pionera mundial en el desarrollo de sistemas probabilistas de predicción basados en técnicas por conjuntos, en particular la técnica bred vectors (cap. 16 en la página 243). Trabaja actualmente en la Universidad de Maryland, trabajó en el NCEP y ha visitado Agencia Estatal de Meteorología (AEMET) en algunas ocasiones como científica asesora para el proyecto AEMET-SREPS.

TIM PALMER, físico-matemático británico, experto en predecibilidad del tiempo y el clima, pionero mundial en el desarrollo de sistemas probabilistas de predicción basados en técnicas por conjuntos, aplicados tanto para la predicción del tiempo como para proyecciones del clima. Ha trabajado en el ECMWF. Actualmente en la Universidad de Oxford.

FRANCO MOLTEnI, experto en predecibilidad del tiempo y el clima, ha trabajado en el Abdus Salam International Centre for Theoretical Physics en Trieste, así como en el ECMWF. Experto internacional en predicción estacional y proyecciones del clima. Junto con PALMER y BuIzZA, es considerado uno de los padres del ECENS.

ROBERTO BUIZZA, experto en predecibilidad del tiempo y el clima, es uno de los directores científicos en el ECMWF. Experto internacional en predicción por conjuntos, fundador de la técnica de los singular vectors (sec. 16.2.6 en la página 249). Junto con PALMER y MOLTENI, es considerado uno de los padres del ECENS.

ZOLTAN TOTH, experto en predicción por conjuntos y temas afines, dirige actividades de investigación y desarrollo en asimilación de datos y SPC, aplicaciones en tiempo real, posproceso, observaciones y verificación en el NCEP.

Figura 26.2: Pioneros en el mundo de los SPC. 


\subsection{Referencias}

[1] Berner, J. y col. "A Spectral Stochastic Kinetic Energy Backscatter Scheme and Its Impact on Flow-Dependent Predictability in the ECMWF Ensemble Prediction System". En: Journal of the Atmospheric Sciences 66.3 (mar. de 2009), páginas 603-626. ISSN: 00224928. DOI: $10.1175 / 2008 J A S 2677.1$ (citado en páginas 389,391 ).

[2] Bishop, Craig H., Etherton, Brian J y MAJumdar, Sharanya J. "Adaptive sampling with the ensemble transform Kalman filter. Part I: Theoretical aspects". En: Monthly weather review 129.3 (2001), páginas 420-436 (citado en página 389).

[3] Bonavita, Massimo y col. "EnKF and Hybrid Gain Ensemble Data Assimilation. Part II: EnKF and Hybrid Gain Results". En: Monthly Weather Review 143.12 (dic. de 2015), páginas 4865-4882. ISSN: 0027-0644. DOI: $10.1175 /$ MWR-D-15-0071.1 (citado en página 389 ).

[4] Buizza, Roberto, Leutbecher, Martin y ISAKSEN, Lars. "Potential use of an ensemble of analyses in the ECMWF Ensemble Prediction System”. En: Quarterly Journal of the Royal Meteorological Society 134.637 (2008), páginas 2051-2066. DOI: 10.1002/ qj 346 (citado en página 389).

[5] BuizzA, Roberto, Miller, Martin J y PALMER, Tim N. "Stochastic representation of model uncertainties in the ECMWF ensemble prediction system". En: Quarterly Journal of the Royal Meteorological Society 125.560 (ago. de 1999), páginas 2887-2908. ISSN: 00359009. DOI: 10 . $1002 / \mathrm{qj}$. 49712556006 (citado en páginas 389, 391).

[6] EbisuZaki, W y Kalnay, Eugenia. "Ensemble experiments with a new lagged average forecasting scheme". En: WMO Research Activities in Atmospheric and Oceanic Modeling Rep 15 (1991), página 308 (citado en página 392).

[7] Epstein, Edward S. "Stochastic dynamic prediction”. En: Tellus 21.6 (1969), pági- nas 739-759. DOI: 10 . 3402 / tellusa . v21i6. 10143 (citado en página 388).

[8] Frogner, Inger-Lise y IVERSEn, Trond. "High-resolution limited-area ensemble predictions based on low-resolution targeted singular vectors". En: Quarterly Journal of the Royal Meteorological Society 128.582 (2002), páginas 1321-1341. DOI: $10.1256 / 003590002320373319$ (citado en página 395).

[9] García-Moya, José Antonio y col. "Predictability of short-range forecasting: A multimodel approach". En: Tellus, Series A: Dynamic Meteorology and Oceanography 63.3 (mayo de 2011), páginas 550-563. ISSN: 02806495. DOI: $10.1111 / \mathrm{j} .1600-0870$. 2010.00506.x (citado en página 395).

[10] Gebhardt, C. y col. "Uncertainties in COSMO-DE precipitation forecasts introduced by model perturbations and variation of lateral boundaries". En: Atmospheric Research 100.2-3 (mayo de 2011), páginas 168-177. ISSN: 0169-8095. DOI: 10 . 1016/J . ATMOSRES . 2010 . 12.008 (citado en página 395).

[11] HÁGEL, Edit y HoRÁnYI, András. "The ARPEGE/ALADIN limited area ensemble prediction system: the impact of global targeted singular vectors". En: Meteorologische Zeitschrift 16.6 (2007), páginas 653-663 (citado en página 395).

[12] Hagelin, Susanna y col. "The Met Office convective-scale ensemble, MOGREPSUK”. En: Quarterly Journal of the Royal Meteorological Society 143.708 (2017), páginas 2846-2861. DOI: 10.1002/qj . 3135 (citado en página 395).

[13] Hoffman, Ross N y Kalnay, Eugenia. "Lagged average forecasting, an alternative to Monte Carlo forecasting". En: Tellus A 35.2 (1983), páginas 100-118. DOI: 10 . 3402/tellusa . v35i2 . 11425 (citado en página 392).

[14] Houtekamer, P.L. y Mitchell, Herschel L. "Ensemble Kalman filtering". En: 
Quarterly Journal of the Royal Meteorological Society 131.613 (oct. de 2005), páginas 3269-3289. ISSN: 00359009. DOI: 10 . 1256/qj . 05.135 (citado en página 389).

[15] ISAKSEN, LARS y col. "The new ensemble of data assimilations". En: ECMWF Newsletter 123 (2010), páginas 17-21 (citado en páginas 388,391$)$.

[16] ISAKSEN, Lars y col. Ensemble of data assimilations at ECMWF. European Centre for Medium-Range Weather Forecasts, 2010 (citado en páginas $388,389,391$ ).

[17] IVERSEN, Trond y col. "Evaluation of 'GLAMEPS'-a proposed multimodel EPS for short range forecasting". En: Tellus, Series A: Dynamic Meteorology and Oceanography 63.3 (mayo de 2011), páginas 513-530. ISSN: 02806495. DOI: 10 . $1111 / j .1600-0870.2010 .00507$.x (citado en página 395$)$.

[18] LeITH, C E. "Theoretical skill of Monte Carlo forecasts". En: Monthly Weather Review 102.6 (1974), páginas 409-418 (citado en página 388).

[19] LORENZ, Edward N. "Empirical orthogonal functions and statistical weather prediction". En: (1956) (citado en página 393).

[20] Lu, Chungu y col. "Short-Range Numerical Weather Prediction Using Time-Lagged Ensembles". En: Weather and Forecasting 22.3 (jun. de 2007), páginas 580-595. ISSN: 08828156. DOI: $10.1175 /$ WAF999. 1 (citado en página 392).

[21] MendonçA, Antônio Marcos y Bonatti, JoséPaulo. "Experiments with EOF-based perturbation methods and their impact on the CPTEC/INPE ensemble prediction system".
En: Monthly Weather Review 137.4 (2009), páginas 1438-1459 (citado en páginas 389, 393).

[22] Molteni, F. y col. "The ECMWF ensemble prediction system: Methodology and validation". En: Quarterly Journal of the Royal Meteorological Society 122.529 (ene. de 1996), páginas 73-119. ISSN: 1477-870X. DOI: 10.1002 / qj . 49712252905 (citado en páginas 389,391 ).

[23] PAlmer, T N y col. "Stochastic parametrization and model uncertainty". En: ECMWF Tech. Memo 598 (2009), páginas 1-42 (citado en página 389).

[24] Toth, Zoltan y KalnAY, Eugenia. "Ensemble forecasting at NMC: The generation of perturbations". En: Bulletin of the american meteorological society 74.12 (1993), páginas 2317-2330 (citado en página 391).

[25] Toth, Zoltan y KaLnAy, Eugenia. "Ensemble forecasting at NCEP and the breeding method". En: Monthly Weather Review 125.12 (1997), páginas 3297-3319 (citado en páginas 389, 391, 395).

[26] WANG, Xuguang y Bishop, Craig H. "A comparison of breeding and ensemble transform Kalman filter ensemble forecast schemes". En: Journal of the atmospheric sciences 60.9 (2003), páginas 1140-1158 (citado en páginas 389, 391).

[27] WEI, Mozheng y col. "Initial perturbations based on the ensemble transform (ET) technique in the NCEP global operational forecast system". En: Tellus A: Dynamic Meteorology and Oceanography 60.1 (ene. de 2008), páginas 62-79. ISSN: 1600-0870. DOI: $10.1111 / \mathrm{j} .1600-0870.2007 .00273 . \mathrm{x}$ (citado en página 389). 
